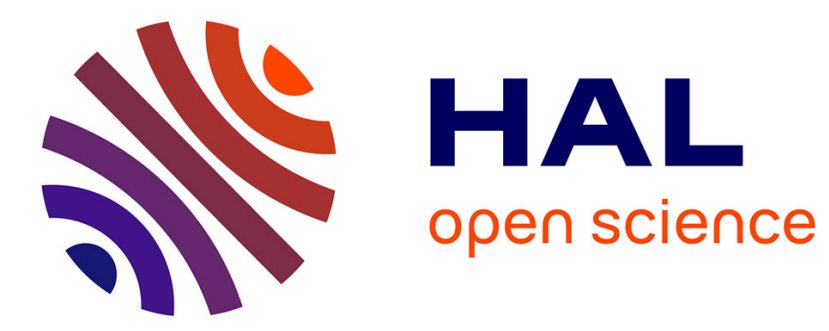

\title{
Faculty Development for FLOSS Education
}

\author{
Becka Morgan, Gregory W. Hislop, Heidi Ellis
}

\section{To cite this version:}

Becka Morgan, Gregory W. Hislop, Heidi Ellis. Faculty Development for FLOSS Education. 15th IFIP International Conference on Open Source Systems (OSS), May 2019, Montreal, QC, Canada. pp.165-171, 10.1007/978-3-030-20883-7_15 . hal-02305709

\section{HAL Id: hal-02305709 \\ https://hal.inria.fr/hal-02305709}

Submitted on 4 Oct 2019

HAL is a multi-disciplinary open access archive for the deposit and dissemination of scientific research documents, whether they are published or not. The documents may come from teaching and research institutions in France or abroad, or from public or private research centers.
L'archive ouverte pluridisciplinaire HAL, est destinée au dépôt et à la diffusion de documents scientifiques de niveau recherche, publiés ou non, émanant des établissements d'enseignement et de recherche français ou étrangers, des laboratoires publics ou privés. 


\title{
Faculty Development for FLOSS Education
}

\author{
Becka Morgan ${ }^{1}$, Gregory W. Hislop ${ }^{2}$, and Heidi J.C. Ellis ${ }^{3}$ \\ ${ }^{1}$ Western Oregon University, Monmouth, Oregon, USA \\ morganb@wou.edu禺 \\ ${ }^{2}$ Drexel University, Philadelphia, PA, USA \\ hislop@drexel.edu \\ ${ }^{3}$ Western New England University, Springfield, MA, USA \\ ellis@wne.edu
}

\begin{abstract}
With the recent upsurge in the development, use, and adoption of free/libre open source software (FLOSS) across all sectors of business, it is critical that graduates of computing degree programs gain an understanding of FLOSS development tools, processes, and culture. However, many faculty members are not fluent in FLOSS development and have little experience in teaching FLOSS. This paper reports on a faculty development program designed to bring instructors up to speed on how to support student learning within FLOSS projects. The paper discusses the challenges to FLOSS education from the instructor's perspective, describes the Professors' Open Source Software Experience (POSSE) workshop, and presents the results of a study into the impact of POSSE on instructors based on semi-structured interviews. This work is part of a larger study into instructor experiences when incorporating Humanitarian Free Open Source Software (HFOSS) into their curriculum.
\end{abstract}

Keywords: Faculty development · FLOSS education.

\section{Introduction}

Free/libre open source software (FLOSS) is rapidly becoming mainstream in industry today as evidenced by Microsoft's acquisition of GitHub, IBM's purchase of Red Hat, and the wide adoption of FLOSS by the majority of enterprise, mid-market, and small businesses [1]. Results of recent surveys indicate that $92 \%$ of respondents identified that their company's applications contain open source libraries [2] and over 50\% of respondents belong to an organization that has an open source software program or has plans to establish one [3].

One reason for the increase in adoption of FLOSS is that it is the source of significant innovation for businesses. Many emerging technologies and approaches that are modernizing computing including cloud computing, containerization, and serverless computing originated with FLOSS. Examples include tools such as Docker and Kubernetes, and FLOSS frameworks such as node.js and Junit. Indeed processes such as continuous integration and DevOps are heavily based on FLOSS concepts.

This increasing use and development of FLOSS creates a clear need for more software professionals who can develop open source software, as well as providing a strong motivation for students to gain an understanding of FLOSS tools and practices as a part of their undergraduate degree programs. Indeed, FLOSS experience can give students 
an edge in the hiring process [4]. However, many instructors are not fluent in FLOSS. This paper discusses an assessment of a professional development program for instructors intended to bring them up to speed in FLOSS culture and tools.

\section{Challenges}

FLOSS practices and tools are not included in typical computing undergraduate degree requirements. In the ACM Curriculum Guidelines, the term "open source" is mentioned only twice in the CS2013 Computer Science Curricula [5] and twice in the IT2017 Information Technology Curricula [6]. The SE2014 Software Engineering Curricula [7] identifies the need for student understanding of FLOSS, yet does not require coverage of FLOSS in the guidelines. This lack of emphasis on FLOSS in professional computing curricula creates a barrier to including FLOSS in the classroom.

Instructors who desire to educate students in FLOSS projects face several additional hurdles. First, many instructors are not familiar with FLOSS culture or tools and face steep learning curves for these, as well as for the FLOSS project domain knowledge. Instructors face an additional learning curve related to how to support student participation in FLOSS projects. Assignment creation, assessment, and development of rubrics are all challenges in the environment of an active FLOSS project and must be undertaken within the constraints of student privacy and intellectual property issues.

Instructors need to learn how to support student learning in the non-traditional environment of a FLOSS project including helping students adjust to a less-structured learning environment and one that has significant size and complexity. Instructors also need to help students with the potentially steep learning curves of FLOSS culture and technologies. Student expectations about the role of the faculty member as a "guide by the side" rather than the "sage on the stage" must be set early and frequently reinforced. Instructors may also need to serve as a liaison between their students and the FLOSS community, which may take additional time.

Another challenge faced by instructors is the selection of an appropriate FLOSS project for use in a course [13]. Ideal FLOSS projects are open to student contributions, welcoming, and have clear communication channels, all while also fulfilling course learning objectives. Several efforts are investigating this problem [8-11].

The FLOSS project environment presents additional challenges. The FLOSS project schedule and instructor's academic calendar may not align well. The ability of a FLOSS project to quickly make major changes in direction may also impact student participation. In addition, instructors must plan for contributions over the limited timespan of a term, typically 10 or 15 weeks, once or twice a year.

Results of a research effort [12] that surveyed faculty members as to the challenges faced when involving students in FLOSS projects indicated that lack of time to prepare course materials was the largest hurdle to student involvement, with lack of time in the course for FLOSS as a close second. Additional hurdles included lack of an appropriate course, difficulty in installing the FLOSS environment, and lack of comfort with the FLOSS learning materials. 


\section{Professors' Open Source Software Experience Workshops}

The faculty development program used in the study is the Professors' Open Source Software Experience (POSSE) workshop. POSSE was originated by Red Hat in 2009, with the goal to help instructors learn about FLOSS so that they can incorporate it into their courses.

In 2013, a team of instructors received National Science Foundation funding to update POSSE contents to include information on pedagogical and curricular considerations, and to provide funding for faculty attendance. In addition, the focus of POSSE was shifted to Humanitarian Free and Open Source Software (HFOSS) projects as there is some evidence that humanitarian projects are more attractive to women and other under-represented groups. A typical POSSE involves 15-25 instructors new to FLOSS and 3-6 workshop facilitators. POSSE workshops are held in three stages. Stage 1 consists of 8 weeks of online learning activities and three IRC meetings are held to answer questions. Stage 2 is a 2.5 day face-to-face meeting held after stage 1 where instructors learn more about FLOSS culture and gain further understanding of how to assess HFOSS projects and incorporate them into their classes. Stage 3 of POSSE occurs after stage 2 and involves instructors working collaboratively on either common projects or common courses to support student involvement in HFOSS. POSSE is supported by over 100 learning materials that instructors may use, located on foss 2 serve.org. Further information on POSSE is contained in [14] and foss2serve.org website.

\section{$4 \quad$ The Study}

POSSE workshops are showing promise for expanding the number of instructors able to support student learning within HFOSS projects and several publications have resulted from POSSE alumni teaching efforts [15-18]. As of early 2019, over 150 instructors from over 120 different academic institutions have completed the workshop. These instructors served as the population for a study that involved an online survey followed by a semi-structured interview with the goal of determining the impact of supporting student participation in HFOSS projects on instructors. In this paper, we discuss the impact of POSSE on instructors as observed in interview results.

The participants in the study were selected from instructors who had participated in a POSSE workshop at least one year prior to the study start in order to focus on instructors who had had time to incorporate HFOSS into their classroom. Out of 77 eligible participants, the group was reduced to 41 by selecting only one instructor per academic institution and focusing on participants who were less experienced in FLOSS education at the time of POSSE attendance. The group included instructors who had been successful and unsuccessful in supporting student participation in HFOSS.

Twenty-six instructors completed the survey and 24 of these agreed to participate in the study. Participants were relatively gender balanced, consisting of 11 women and 13 men and participants came from both 2 and 4-year institutions located in 16 different U.S. states. Further details on the study design may be found in [12]. 
The semi-structured interview included questions such as "What was the biggest hurdle that you encountered and how did you overcome it?" and "What have you learned from working with a FLOSS community?" The study resulted in a considerable volume of data, which is still being analyzed. The focus of this paper is on the impact of POSSE workshops on instructors.

\subsection{Results}

The corpus of the 24 semi-structured instructor interviews was searched for all occurrences of the terms "POSSE", "workshop*", "roundup" and "meet*". The occurrences of these terms within their context were analyzed by two authors who coded the answers and organized them into categories. The coding process was performed independently to avoid bias and a conflict resolution meeting was used to come to agreement on the categories. Five main themes, described below, emerged.

Helpfulness. Ten of the respondents identified the POSSE workshop has being helpful using phrases such as "learning activities were immensely helpful", "very valuable", and "eye-opening". One participant stated, "POSSE experience forever changed my perception of what it means to be open source..." An additional three respondents indicated that they had gained a better understanding of FLOSS tools. This feedback clearly indicates that instructors feel that they gain useful knowledge and skills from the POSSE workshop and that the workshop is helpful in supporting student participation in HFOSS projects.

Materials. Nine respondents indicated that they used the POSSE workshop materials in their courses, either directly or with modification. One participant indicated that POSSE was "...quite helpful with learning activities, I got good ideas for activities..." These responses support the observation that POSSE workshops are fulfilling their goal of providing instructors with the skills and materials they need to support student learning within HFOSS projects.

Enhancements. Another major theme that emerged was possible enhancements to the POSSE workshop. Ten participants identified augmentations to POSSE that they thought would help them support student participation in HFOSS. These included:

- Longer workshop meeting with additional hands-on experience with projects

- Tutorials that instructors and students could use to learn tools

- List of approachable HFOSS projects including current community contacts

- Repository of exemplars of student participation in HFOSS

- Curriculum in a box where a project and set of tools are packaged in a VM

- More advanced workshop that builds on current POSSE

Community. One recurrent theme throughout most of the interviews was the value provided by the POSSE community and the need for it to be an ongoing and healthy community. The use of POSSE "Roundups" and sprints where instructors meet faceto-face to share ideas and work on activities was clearly valued. One respondent indicated "... round ups for me are very beneficial ... so I can touch base with other people and hear what other people are doing and exchange ideas." In addition, some respondents indicated that attending a second POSSE workshop was or would be helpful. Clearly POSSE participants value the community established by the workshops and 
desire to see that community continue and grow. POSSE appears to work well as an introduction to the topic and the community, but there needs to be ongoing support for the community.

Shortcomings. Another important theme that emerged from the analysis was identification of the shortcomings of the POSSE experience. Several respondents indicated frustration with not being able to get the HFOSS project development environment installed. One respondent who tried to get an HFOSS project installed while attending POSSE stated, "We spend all of our working time trying to set up the system and never got it set up. That was a kind of negative experience on my part." Another respondent indicated that the activities in stage 1 and stage 2 needed to be more tightly related stating, "We had a series of HWs prior to workshop, then we didn't even really discuss them at the workshop..." Another respondent indicated that providing each participant with a concrete take-away appropriate for their class would have been helpful.

Overall, the observations about POSSE indicate that participants are gaining knowledge and skills from the workshops. The majority of participants indicated that they found the workshop helpful and that they used workshop materials in their teaching. The need for continuing community was also a resonant thread throughout the interviews. One respondent noted, "most important takeaway for me is the desire for $<$ the $>$ POSSE organism to remain alive and vibrant moving forward". Feedback on the shortcomings of the workshop focuses on identification of appropriate course materials and the availability of approachable and tractable HFOSS projects. Instructors involved in carrying out POSSE are currently working to address the shortcomings.

\subsection{Limitations}

The biggest limitation of the study is that the participants in the study self-selected to participate in the POSSE workshop. A second limitation is the independent coding of the responses. Lastly, the number of professors involved is relatively low, which may limit the generalization of the results.

\section{$5 \quad$ Conclusion and Future Work}

The work presented in this paper is a part of a larger study into the impact of student participation in HFOSS on instructors. Results of this study will provide insight into how to increase the number of instructors in the FLOSS education community to fulfill the larger goal of increasing the number of students who graduate ready to contribute to FLOSS projects. Overall, results of analyzing instructor comments on the POSSE workshop experience are positive and appear to indicate that instructors are better able to support student participation in the classroom. The POSSE team is working to address the identified shortcomings of difficulty in project installation and identification of appropriate assignments for those instructors first learning to support FLOSS education. Future areas of study include analysis of instructor expectation, analysis of instructor factors that lead to student success, and how FLOSS education impacts instructor self-perception as a teacher. 


\section{References}

1. 10th Anniversary of the Open Source Survey, 2016, www.northbridge. com/2016-future-open-source-survey-results Last accessed 11 Jan 2019

2. "How to make open source work better for everyone, 9 key insights from the 2018 Tidelift professional open source survey" July, 2018, https://tidelift.com/about/2018-Tidelift-professional-open-source-survey-results Last accessed 20 Dec 2018

3. Hecht, L., and Clark, L., "Survey: Open Source Programs Are a Best Practice Among Large Companies," 30 Aug 2018, https://thenewstack.io/survey-open-source-programs-are-a-best-practice-among-largecompanies/ Last accessed 11 Jan 2019

4. Hansson, D.H.: Reduce the risk, hire from open source, https://dhh.dk//arc/2005_09.html Last accessed 13 Jan 2019

5. Computer Science Curricula 2013 Curriculum Guidelines for Undergraduate Degree Programs in Computer Science, December 20, 2013 https://www.acm.org/binaries/content/assets/education/cs2013 web final.pdf Last accessed 11 Jan 2019

6. Information Technology Curricula 2017 IT2017 Curriculum Guidelines for Baccalaureate Degree Programs in Information Technology, December 10, 2017 https://www.acm.org/binaries/content/assets/education/curricula-recommendations/it2017.pdf Last accessed 11 Jan 2019

7. Software Engineering 2014 Curriculum Guidelines for Undergraduate Degree Programs in Software Engineering, 23 Feb 2015

https://www.acm.org/binaries/content/assets/education/se2014.pdf Last accessed 11 Jan 2019

8. Gokhale, S. D., Smith, T.M., McCartney, R.: Integrating open source software into software engineering curriculum: challenges in selecting projects. In: Proceedings of the First International Workshop on Software Engineering Education Based on Real-World Experiences (EduRex '12), pp. 9-12. IEEE Press, Piscataway, NJ, USA (2012)

9. Smith, T.M., McCartney, R., Gokhale, S.S., Kaczmarczyk, L.C.: Selecting open source software projects to teach software engineering. In: Proceedings of the 45th ACM technical symposium on Computer science education (SIGCSE '14), pp. 397-402, ACM, New York, NY, USA, (2014). DOI=10.1145/2538862.2538932 http://doi.acm.org/10.1145/2538862.2538932

10. Ellis, H.J.C., Hislop, G.W., Burdge, D.: Courseware: HFOSS Project Evaluation. In: Proceedings of the 2017 ACM Conference on Innovation and Technology in Computer Science Education (ITiCSE '17). ACM, New York, NY, USA, 90-91. (2017) DOI: https://doi.org/10.1145/3059009.3072975

11. Ellis, H.J.C., Hislop, G.W., Purcell, M.: Project selection for student involvement in humanitarian FOSS, In: 26th International Conference on Software Engineering Education and Training (CSEE\&T), San Francisco, CA, pp. 359-361, (2013) doi:10.1109/CSEET.2013.6595279

12. Postner, L., Ellis, H.J.C., Hislop, G.W.: A Survey of Instructors' Experiences Supporting Student Learning using HFOSS Projects, In: Proceedings of the 49th ACM Technical Symposium on Computer Science Education (SIGCSE '18), pp. 203-208, ACM, New York, NY, USA, 203-208. DOI: https://doi.org/10.1145/3159450.3159524 (2018)

13. Pinto, G.H.L., Filho, F.F., Steinmacher, I., Gerosa, M.A.:Training Software Engineers Using Open-Source Software: The Professors' Perspective, In: 2017 IEEE 30th Conference on Software Engineering Education and Training (CSEET), pp. 117-121, doi=10.1109/CSEET.2017.27, (2017) 
14. Ellis HJC, Chua M, Hislop GW, Purcell M, Dziallas S. Towards a model of faculty development for FOSS in education. In: 2013 26th International Conference on Software Engineering Education and Training (CSEE\&T) (may 2013); 2013. p. 269-73.

15. Braught, G. et. al.: A Multi-Institutional Perspective on H/FOSS Projects in the Computing Curriculum, ACM Trans. Comput. Educ. 18, 2, Article 7, pp. 7:1 - 7:31, (2018). DOI: https://doi.org/10.1145/3145476

16. Crain, S.P.: Open source security assessment as a class project, In: J. Comput. Sci. Coll, 32, 6, pp. 41-53, 2017

17. Murphy, C., Buffardi, K., Dehlinger, J., Lambert, L., Veilleux, N.: Community Engagement with Free and Open Source Software, In: Proceedings of the 2017 ACM SIGCSE Technical Symposium on Computer Science Education (SIGCSE '17), pp. 669-670, ACM, New York, NY, USA, 669-670. (2017) DOI: https://doi.org/10.1145/3017680.3017682

18. Buffardi, K.: Localized open source collaboration in software engineering education, In: 2015 IEEE Frontiers in Education Conference (FIE), El Paso, TX, 2015, pp. 1-5 (2015), doi: 10.1109/FIE.2015.7344142 